Leukemia - Lymphoma and Myeloma

\title{
Tandem Autologous Hematopoietic Stem Cell Transplantation in Very Young Patients with Multiple Myeloma
}

\author{
Rahul Naithani ${ }^{1} \quad$ Nitin Dayal ${ }^{2}$ Reeta Rai ${ }^{3}$ \\ ${ }^{1}$ Division of Hematology \& Bone Marrow Transplantation, Max \\ Super Specialty Hospital, Saket, New Delhi, India \\ 2Department of Lab Medicine, Max Super Specialty Hospital, Saket, \\ New Delhi, India \\ ${ }^{3}$ Department of Transfusion Medicine, Max Super Specialty \\ Hospital, Saket, New Delhi, India
}

\section{South Asian J Cancer 2021;9:233-235.}

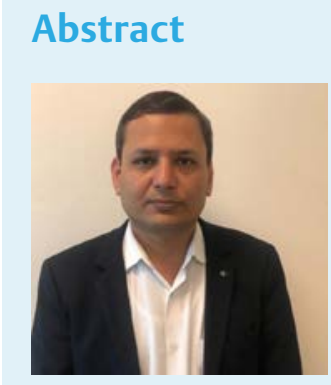

Rahul Naithani, DM
Keywords

- autologous

- tandem transplant

- very young myeloma
Introduction Multiple myeloma (MM) in very young patients is uncommon, and no treatment guidelines exist for these patients.

Patients and Methods We performed a retrospective analysis of five very young myeloma patients who underwent tandem autologous hematopoietic stem cell transplantation (HSCT).

Results The median age was 37 years (range $=34-40$ years). A median of two leukapheresis was performed (range $=1-4$ ). The median number of hematopoietic stem cells collected was $5.4 \times 10^{6} / \mathrm{kg}\left(4.4-8.2 \times 10^{6} / \mathrm{kg}\right)$. During first transplant, four patients received melphalan of $200 \mathrm{mg} / \mathrm{m}^{2}$ and one patient received melphalan of $140 \mathrm{mg} / \mathrm{m}^{2}$ (due to renal failure) as conditioning regimen. Second transplant conditioning was melphalan of $200 \mathrm{mg} / \mathrm{m}^{2}$ for one patient and melphalan of $140 \mathrm{mg} / \mathrm{m}^{2}$ for remaining four patients. Two patients were in complete remission, and two were in very good partial remission and one patient progressed to active disease at the time of tandem autologous bone marrow transplant. All patients developed significant mucositis. Neutrophil and platelet recovery was longer in tandem autologous hematopoietic stem cell transplant. More viral infections were seen in tandem transplant. Day 30 and day 100 mortality was nil.

Conclusion We present data on tandem autologous HSCTs in very young patients with MM in India. Responses continued to improve in this small series.

\section{Introduction}

Outcome of patients with multiple myeloma (MM) has improved with bortezomib and immunomodulatory drug combinations. ${ }^{1}$ However, relapse is the rule and median progression free survival (PFS) after first-line therapy is usually 4 years. ${ }^{2}$ It has been very recently shown that even in the era

\section{DOI https://doi.org/10.1055/s-0041-1727068 ISSN 2278-330X}

How to cite this article: Naithani R, Dayal N, Rai R. Tandem autologous hematopoietic stem cell transplantation in very young patients with multiple myeloma. South Asian J Cancer 2021;9(4):233-235. of novel agents, high-dose melphalan and autologous hematopoietic stem cell transplant (ASCT) result in higher remission rates and prolonged PFS in patients with MM. ${ }^{3}$ Published data on myeloma in very young ( $<40$ years of age) are rare and treatment guidelines in this subgroup are not well defined. ${ }^{4}$ It is unclear if very young MM patients should be treated differently as a PFS of 4 years is certainly not acceptable in this

(c) 2021. MedIntel Services Pvt Ltd.

This is an open access article published by Thieme under the terms of the Creative Commons Attribution-NonDerivative-NonCommercial-License, permitting copying and reproduction so long as the original work is given appropriate credit. Contents may not be used for commercial purposes, or adapted, remixed, transformed or built upon. (https://creativecommons.org/licenses/by-nc-nd/4.0/).

Thieme Medical and Scientific Publishers Private Ltd A-12, Second Floor, Sector -2, NOIDA -201301, India 
subgroup and if this constitutes a high-risk group in this regard. A recent meta-analysis has showed superiority of tandem hematopoietic stem cell transplantation (HSCT) in high-risk MM even in the era of novel agents. ${ }^{5}$ There are no published data on the safety and effectiveness of tandem ASCT in very young myeloma patients. Recently, Jungova et al have demonstrated significantly better treatment-free survival and overall survival in the group where tandem ASCT was performed in the Czech Republic. ${ }^{6}$ We share our experience of tandem ASCTs in very young MM patients.

\section{Patients and Methods}

Peripheral blood stem cell apheresis was performed by using hemonetics MCS plus or Cobe Spectra apheresis machine. Stem cell yield of the harvest was determined by CD34 cell enumeration using the ISHAGE protocol. ${ }^{7}$ Hematopoietic stem cells were cryopreserved in dimethyl sulfoxide at $-80^{\circ} \mathrm{C}$ in blood bank refrigerator without controlled-rate freezing. HDM was administered on same day evening when adequate stem cell dose was collected. Stem cells were infused intravenously on day 0 generally 24 hours after the completion of melphalan. Patients received granulocyte-colony stimulating factor (G-CSF) $300 \mu \mathrm{g}$ subcutaneous once a day starting on day +6 after infusion of stem cells until the time of engraftment.

\section{Results}

Sixty patients underwent autologous HSCT at our center. Data on five patients who received tandem ASCT are presented here. Four patients received planned tandem HSCT while one patient received it as rescue measure (explained later). Median age was 37 years (range $=34-40$ years). There were four males and one female. Fluorescence in situ hybridization for MM was sent in three patients and was negative for common six abnormalities. Two patients were mobilized with G-CSF, two with cyclophosphamide-G-CSF, and in one patient, hematopoietic stem cells (HSCs) were collected while recovery from DT PACE chemotherapy. A median of two leukapheresis was performed (range $=1-4$ ). Median number of HSCs collected was $5.4 \times 10^{6} / \mathrm{kg}\left(4.4-8.2 \times 10^{6} / \mathrm{kg}\right)$. Half dose was used for first transplant and half-dose was cryopreserved for tandem ASCT.

Three patients received a second tandem ASCT after 3 months and two patients received it after 6 months. During the first transplant, four patients received melphalan of 200 $\mathrm{mg} / \mathrm{m}^{2}$ and one patient received melphalan of $140 \mathrm{mg} / \mathrm{m}^{2}$ (due to renal failure) as conditioning regimen. Second transplant conditioning was melphalan of $200 \mathrm{mg} / \mathrm{m}^{2}$ for one patient and melphalan of $140 \mathrm{mg} / \mathrm{m}^{2}$ for the remaining four patients.

\section{First Transplant}

Two patients were in complete remission (CR) and three were in partial remission (PR) at the time of first autologous bone marrow transplant (BMT). Median HSCs infused were $3.10^{6} / \mathrm{kg}\left(2.4-4.2 \times 10^{6} / \mathrm{kg}\right)$. The median time to neutrophil and platelet engraftment was 11 days (10-12 days). Four patients developed fever lasting 1 to 5 days. One patient had urinary symptoms and another had Staphylococcus epidermidis in blood culture. All patients developed mucositis grade III (II-IV). One patient developed Evans syndrome after engraftment and another patient developed catheter-associated deep-venous thrombosis. Median hospitalization duration was 19 days (16-25 days).

\section{Tandem Autologous Hematopoietic Stem Cell Transplant}

Two patients were in CR and two were in very good PR (VGPR) and one patient progressed to active disease at the time of tandem autologous BMT. Median cryopreserved HSCs infused were $2.6 \times 10^{6} / \mathrm{kg}\left(2-4 \times 10^{6} / \mathrm{kg}\right)$. Cell dose was calculated at the time of cryopreservation and not during infusion when the products were thawed. All patients developed mucositis grade II (II-III). The median time to neutrophil engraftment was 13 days (12-15 days). The median time to platelet engraftment was 14 days (12-17 days). Four patients developed fever. One patient developed Escherichia coli in blood culture, another had Staphylococcus hemolyticus followed by Pseudomonas aeruginosa (carbapenem resistant) in blood. One patient had herpes mucositis. One patient developed cytomegalovirus reactivation during follow-up. Median hospitalization duration was 18 days (15-22 days).

Patients in CR continue to remain in CR till date. Two VGPR patients converted to CR after tandem ASCT.

Day 30 and day 100 treatment-related mortality was nil. One patient died 16 months after second of tandem ASCT with dengue fever and multiorgan dysfunction. She presented 1 week late to hospital emergency with renal failure and could not be revived. She was in CR at that time. Another 39-year-old male had extensive extramedullary disease at presentation itself. He did not respond to initial therapy and was salvaged with Dexamethasone $+/$ - thalidomide with infusion of cisplatin, doxorubicin, cyclophosphamide. and etoposide (DTPACE) chemotherapy with which he achieved PR. He progressed to active disease at the time of tandem autologous BMT. Intent was changed to palliation. He developed significant cytopenia requiring transfusion support after palliative radiation therapy. Since his counts were not recovering, it was decided to salvage his marrow with cryopreserved HSCs. Since stem cells were to be infused, it was discussed with family and melphalan was also administered. He succumbed 7 months later to progressive disease.

Other three patients continue to be in CR at a follow-up of 46.61 and 84 months.

\section{Discussion}

Clinicians across the world are struggling to find best ways of prolonging survival and if possible induce operational cure in MM. Rarity of disease always makes treatment decision-making difficult. The American Society for Blood and Marrow Transplantation guidelines have kept a tandem ASCT as an open question but allows it in special situations. ${ }^{8}$ IFM trial was first to demonstrate a benefit from the tandem ASCT in event-free survival, relapse-free survival (RFS), and OS. Patients achieving less than a very good partial response 
benefited most from the tandem procedure. ${ }^{9}$ However, the Bologna 96 and the HOVON 24 trials showed the tandem arm did not improve OS (because of ability to salvage patients after relapse) but improved event-free survival. ${ }^{10,11}$ Similarly, HOVON-65/GMMGHD4 trial also demonstrated an improvement in PFS in tandem ASCT arm. ${ }^{12}$ Another study had demonstrated noninferiority of single autologous versus tandem autologous BMT. ${ }^{13}$ With the recent meta-analysis documenting superiority of tandem ASCT in high-risk Group V, it may be worth a try in very young myeloma patients.

It is worthwhile to note that the median age in these studies have been much higher. Indian patients are a decade younger. ${ }^{14}$ In Indian setting, PFS is realistically more important than OS. Once patients relapse, often they do not have access to newer salvage medicines which people in western centers have and many a times, they are financially exhausted. Sixty patients have undergone ASCT for myeloma at our center. We documented $84 \%$ OS at a shorted median follow-up in that cohort with single transplant. This contrasts the 5-year follow-up in the present small cohort of very young myeloma patients in this manuscript.

We do not routinely offer tandem ASCT in myeloma. We have shown that tandem ASCT are feasible in very young patients. In fact, responses kept on improving after ASCTs. Patients certainly experienced more adverse effects during tandem ASCT. Neutrophil recovery and platelet recovery was prolonged during tandem ASCT. There were more documented infections including viral infections. One death was attributable to bad biology of disease and another was unrelated. Median hospitalization duration was shorter in tandem transplant as stem cells were already cryopreserved.

A longer follow-up and pooling of data from centers worldwide will make enable us to take wise decisions in these very young patients.

\section{Conclusion}

Tandem autologous HSCTs are feasible and effective in very young MM patients.

Note

Informed consent was obtained from all individual participants included in the study at the time of transplant. The authors certify that they have obtained all appropriate patient consent forms. In the form, the patients have given their consent for their images and other clinical information to be reported in the journal. The patients understand that their names and initials will not be published and due efforts will be made to conceal their identity, but anonymity cannot be guaranteed.

\section{Funding}

None.

Conflict of Interest

None declared.

\section{References}

1 Durie BGM, Hoering A, Abidi MH, et al. Bortezomib with lenalidomide and dexamethasone versus lenalidomide and dexamethasone alone in patients with newly diagnosed myeloma without intent for immediate autologous stem-cell transplant (SWOG S0777): a randomised, open-label, phase 3 trial. Lancet 2017;389(10068):519-527

2 Kastritis E, Zervas K, Symeonidis A, et al. Improved survival of patients with multiple myeloma after the introduction of novel agents and the applicability of the International Staging System (ISS): an analysis of the Greek Myeloma Study Group (GMSG) Leukemia 2009;23(6):1152-1157

3 Attal M, Lauwers-Cances V, Hulin C, et al; IFM 2009 Study. Lenalidomide, bortezomib, and dexamethasone with transplantation for myeloma. NEnglJ Med 2017;376(14):1311-1320

4 Yanamandra U, Saini N, Chauhan P, et al. AYA-myeloma: real-world, single-center experience over last 5 years. J Adolesc Young Adult Oncol 2018;7(1):120-124

5 Dhakal B, Szabo A, Chhabra S, et al. Autologous transplantation for newly diagnosed multiple myeloma in the era of novel agent induction: a systematic review and meta-analysis. JAMA Oncol 2018;4(3):343-350

6 Jungova A, Vokurka S, Schutzova M, et al. Comparison of autologous hematopoietic cell transplantation performed in tandem and in disease relapse in multiple myeloma patients. Neoplasma 2018;65(6):952-957

7 Naithani R, Dayal N, Dixit G. Single versus dual platform analysis for hematopoietic stem cell enumeration using ISHAGE protocol. Indian J Hematol Blood Transfus 2017;33(3):370-374

8 Shah N, Callander N, Ganguly S, et al; American Society for Blood and Marrow Transplantation. Hematopoietic stem cell transplantation for multiple myeloma: Guidelines from the American Society for Blood and Marrow Transplantation. Biol Blood Marrow Transplant 2015;21(7):1155-1166

9 Attal M, Harousseau JL, Facon $\mathrm{T}$, et al; InterGroupe Francophone du Myélome. Single versus double autologous stem-cell transplantation for multiple myeloma. N Engl J Med 2003;349(26):2495-2502

10 Cavo M, Tosi P, Zamagni E, et al. Prospective, randomized study of single compared with double autologous stem-cell transplantation for multiple myeloma: Bologna 96 clinical study. J Clin Oncol 2007;25(17):2434-2441

11 Sonneveld P, van der Holt B, Segeren CM, et al; Dutch-Belgian Hemato-Oncology Cooperative Group (HOVON). Intermediate-dose melphalan compared with myeloablative treatment in multiple myeloma: long-term follow-up of the Dutch Cooperative Group HOVON 24 trial. Haematologica 2007;92(7):928-935

12 Sonneveld P, Schmidt-Wolf IG, van der Holt B, et al. Bortezomib induction and maintenance treatment in patients with newly diagnosed multiple myeloma: results of the randomized phase III HOVON-65/ GMMG-HD4 trial. J Clin Oncol 2012;30(24):2946-2955

13 Mai EK, Benner A, Bertsch U, et al. Single versus tandem high-dose melphalan followed by autologous blood stem cell transplantation in multiple myeloma: long-term results from the phase III GMMG-HD2 trial. Br J Haematol 2016;173(5):731-741

14 Naithani R, Dayal N, Rai R, Pathak S, Singh M. Autologous hematopoietic stem cell transplantation for multiple myeloma in India. Indian J Hematol Blood Transfus 2018;34(3):564-565 